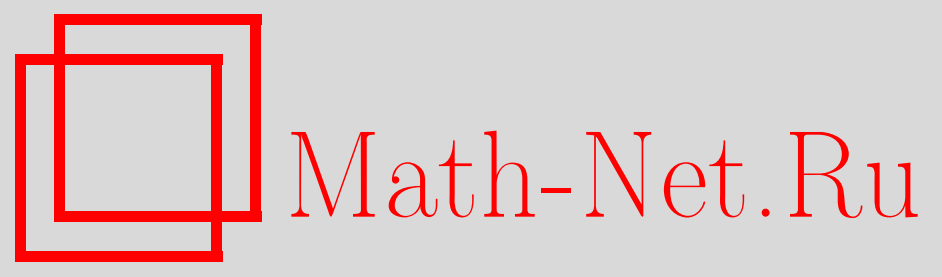

Л. Г. Куракин, В. И. Юдович, Бифуркация коразмерности 1 ответвления двумерных инвариантных торов от семейства равновесий в системах с косимметрией, Матем. заметки, 2003, том 73, выпуск 5, 796-800

DOI: https://doi.org/10.4213/mzm633

Использование Общероссийского математического портала Math-Net.Ru подразумевает, что вы прочитали и согласны с пользовательским соглашением http://www.mathnet.ru/rus/agreement

Параметры загрузки:

IP : 54.81 .137 .203

26 апреля 2023 г., $17: 40: 41$

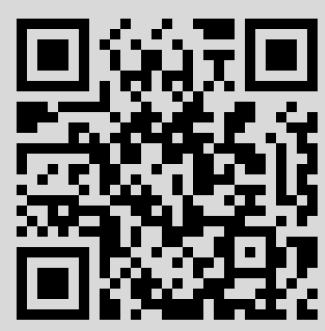




\section{БИФУРКАЦИЯ КОРАЗМЕРНОСТИ 1 ОТВЕТВЛЕНИЯ ДВУМЕРНЫХ ИНВАРИАНТНЫХ ТОРОВ ОТ СЕМЕЙСТВА РАВНОВЕСИЙ В СИСТЕМАХ С КОСИММЕТРИЕЙ}

\section{Л.Г. Куракин, В.И. Юдович}

Непрерьвное однопараметрическое семейство равновесий является обычным объектом для векторных полей с нетривиальной косимметрией. Спектр устойчивости равновесия меняется вдоль такого семейства, но всегда содержит точку нуль. В условиях общего положения семейство состоит из устойчивых и неустойчивых дуг, разделяемых граничными равновесиями, которые нейтрально устойчивы по линейному приближению. Таким образом, наличие в косимметричной системе граничного равновесия со спектром, имеющим нуль и пару ( $k$ пар) чисто мнимых собственных значений есть ситуация общего положения (имеет коразмерность $k-1$ ).

В данной работе исследована бифуркация ответвления инвариантных двумерных торов в системах с косимметрией от граничного равновесия, чей спектр содержит нулевое и две пары чисто мнимых собственных значений. Обнаружен ряд эффектов, новых по сравнению с классическим случаем изолированного равновесия: рассматриваемая бифуркация имеет коразмерность 1 (в случае изолированного равновесия - 2); она не сопровождается бифуркацией ответвления нормального предельного цикла; имеется возможность рождения устойчивой дуги на неустойчивой (случай i) п. 1).

Ссылки по теории косимметрии и применяемым методам центрального многообразия и Ляунова-Шмидта см. в работах [1]-[3], где изучено рождение цикла из семейства равновесий и исследованы бифуркации, сопровождающие монотонную потерю устойчивости равновесия косимметричной системы. Поскольку речь идет о локальной бифуркации, достаточно рассмотреть уравнение на линейном (гильбертовом) пространстве, отождествляя при этом векторы и ковекторы, а косимметрии (пфаффовы формы, аннулирующие данное векторное поле) - с векторными полями.

1. Метод центрального многообразия. Рассмотрим обыкновенное дифференциальное уравнение в гильбертовом пространстве $H$

$$
\dot{\theta}=F(\theta, \lambda)
$$

с векторным параметром $\lambda=\left(\lambda_{1}, \ldots, \lambda_{n}\right) \in \mathbb{R}^{n}$, имеющее равновесие $\theta_{0}$ при некотором $\lambda=\lambda_{0}$, так что $F\left(\theta_{0}, \lambda_{0}\right)=0$. Предположим, что векторное поле $F$ допускает косимметрию $\mathfrak{L}$, зависящую от $\lambda$. Это означает, что задано отображение $\mathfrak{L}: H \times \mathbb{R}^{n} \rightarrow H:(\theta, \lambda) \rightarrow \mathfrak{L}(\theta, \lambda)$ такое, что при любом $\lambda \in \mathbb{R}^{n}$ для всех $\theta \in H$ векторы $F(\theta, \lambda), \mathfrak{L}(\theta, \lambda)$ ортогональны: $(F(\theta, \lambda), \mathfrak{L}(\theta, \lambda))=0$.

Исследование, описанное в данной публикации, стало возможным благодаря частичной поддержке Американского фонда гражданских исследований и развития (АФГИР), грант № RM12084, Российского фонда фундаментальных исследований, гранты № 01-01-22002 и № 02-01-00337 и программы "Университеты России - фундаментальные исследования", проект № УР.04.01.063. 
Примем следующие гипотезы.

H1. Отображение $\mathfrak{L}$ непрерывно, а отображение $F$ аналитично в некоторой окрестности точки $\left(\theta_{0}, \lambda_{0}\right)$.

H2. Равновесие $\theta_{0}$ некосимметрично: $L_{0}=\mathfrak{L}\left(\theta_{0}, \lambda_{0}\right) \neq 0$. Из этого следует, что точка 0 принадлежит спектру $\sigma\left(A_{0}\right)$ производной $A_{0}=F_{\theta}^{\prime}\left(\theta_{0}, \lambda_{0}\right)$. При этом $A_{0}^{*} L_{0}=0$.

Н3. Пересечение спектра $\sigma\left(A_{0}\right)$ с мнимой осью состоит из простых полюсов $\left\{0, \pm i \omega_{1}, \pm i \omega_{2}\right\}$, $\omega_{1}>\omega_{2}>0,-$ общих собственных значений оператора $A_{0}$ и его сопряженного $A_{0}^{*}$. Отсутствуют все резонансы второго и третьего порядков: $1: 2,1: 1,1: 3$, так что $\omega_{1} \neq 2 \omega_{2}, \omega_{1} \neq \omega_{2}, \omega_{1} \neq 3 \omega_{2}$.

Н4. Спектр $\sigma\left(A_{0}\right)$ лежит в замыкании левой полуплоскости.

В рассматриваемой ситуации применима косимметрическая версия теоремы о неявной функции (см. [1]-[3]). Из нее следует, что множество равновесий уравнения (1) локально в окрестности точки $\left(\theta_{0}, \lambda_{0}\right)$, представляет собой $(n+1)$-мерное подмногообразие в $H \times \mathbb{R}^{n}$.

От уравнения (1) переходим к нормализованной системе на центральном многообразии (см. [4]), в которой отбрасываем старшие слагаемые. Полученная система обладает симметрией, что позволяет перейти к ее трехмерной факторсистеме в полупространстве $\left\{\left(x, \rho_{1}, \rho_{2}\right) \in \mathbb{R}^{3}: \rho_{1} \geqslant 0\right.$, $\left.\rho_{2} \geqslant 0\right\}$ :

$$
\dot{x}=a_{1} \rho_{1}+a_{2} \rho_{2}, \quad \dot{\rho}_{k}=\rho_{k}\left(b_{k 1} \rho_{1}+b_{k 2} \rho_{2}+b_{k 3} x+\beta_{k}\right) .
$$

Здесь $a_{k}, b_{k l}$ - вещественные коэффициенты, а $\beta_{k}$ - малые вещественные параметры $(k=1,2$, $l=1,2,3)$. Система (2) имеет семейство равновесий $\Gamma$, задаваемое уравнениями $\rho_{1}=0, \rho_{2}=0$. Предельному тору (циклу) системы (1) отвечает равновесие $\left(x_{*}, \rho_{1 *}, \rho_{2 *}\right)$ системы (2) такое, что $\rho_{1 *}>0, \rho_{2 *}>0\left(\rho_{1 *}>0, \rho_{2 *}=0\right.$ или $\left.\rho_{1 *}=0, \rho_{2 *}>0\right)$. Система (1) не имеет предельных ииклов, если $a_{1} a_{2} \neq 0$.

Предполагая выполнение условий $a_{1} a_{2} b_{13} b_{23} \neq 0, b_{23} b_{12}-b_{22} b_{13} \neq 0$, строим главное семейство векторных полей

$$
\dot{x}=\rho_{1}+\varkappa \rho_{2}, \quad \dot{\rho}_{1}=a x \rho_{1}, \quad \dot{\rho}_{2}=\rho_{2}\left(b \rho_{1}-\rho_{2}+c x+\beta\right),
$$

где $\varkappa$ - дискретный параметр, принимающий два значения: $\varkappa= \pm 1$. Оно получено уменьшением числа непрерывных параметров системы (2) с помощью масштабных замен переменных, обращения времени и еще одной замены, с последующим отбрасыванием несущественных слагаемых.

Внутренние бифуркации на семействе равновесий. Спектр устойчивости равновесия $\left(x_{0}, 0,0\right)$ векторного поля (3) меняется вдоль семейства $\Gamma$ (параметр $\beta$ мал и фиксирован) и имеет вид $\left\{0, \lambda_{1}\right.$, $\left.\lambda_{2}\right\}$, где $\lambda_{1}=a x_{0}, \lambda_{2}=c x_{0}+\beta-$ собственные значения.

Введем обозначения $D_{--}, D_{-+}, D_{+-}, D_{++}$для открытых дуг, образованных на прямой $\Gamma$ равновесиями одного типа, так что $\left(x_{0}, 0,0\right) \in D_{\operatorname{sgn}} \lambda_{1}, \operatorname{sgn} \lambda_{2}$.

Анализ системы (3) приводит к следующим выводам.

Семейство равновесий $\rho_{1}=\rho_{2}=0$ системы (3) разбивается граничными точками $K_{1}=(0,0,0)$ и $K_{2}=(-\beta / c, 0,0)$ на дуги, образованные однотипными равновесиями. Движению вдоль прямой $(x, 0,0)$ в направлении возрастания величины $x$ отвечают последовательности дуг и граничных точек.

i) Cлучай $a>0, c<0: D_{-+} K_{2} D_{--} K_{1} D_{+-}$при $\beta<0$ u $D_{-+} K_{1} D_{++} K_{2} D_{+-}$, когда $\beta>0$.

ii) Cлучай $a>0, c>0: D_{--} K_{1} D_{+-} K_{2} D_{++}$, если $\beta<0$, u $D_{--} K_{2} D_{-+} K_{1} D_{++}$, когда $\beta>0$.

Случаи iii) $a<0, c>0$ и iv) $a<0, c<0$ сводятся к i) и ii) обращением времени $t \mapsto-t$.

Условия существования тора и анализ его спектра устойчивости. В случае $\varkappa=1$ система (3) не имеет равновесий, не лежащих на прямой $Г$. Пусть теперь $\varkappa=-1$. Равновесие системы (3)

$$
x_{*}=0, \quad \rho_{1 *}=\rho_{2 *}=\rho_{*}, \quad \rho_{*}=\frac{\beta}{1-b}
$$


при $\rho_{*}>0$ отвечает тору системы (1). Значит, $\beta=0$ есть точка ответвления тора.

Назовем типом грубого равновесия четверку целых неотрицательных чисел $\left(m_{-}, n_{-}, m_{+}\right.$, $\left.n_{+}\right)$, где $m_{-}$и $n_{-}\left(m_{+}\right.$и $\left.n_{+}\right)$- означают соответственно число вещественных и комплексно-сопряженньх пар собственных значений в левой (правой) полуплоскости.

Равновесие (4) имеет следующий тип:

1) $(1,0,2,0)$, если выполнень оба неравенства $B=a-c>0, C=a(b-1)>0$;

2) $(2,0,1,0)$, когда $B>0, C<0$;

3) $(0,2,1,0)$ в случае $B<0, C<0$;

4) $(1,2,0,0)$, когда $B<0, C>0, D=B+C<0$;

5) $(1,0,0,2)$, если $B<0, D>0$.

2. Метод Ляпунова-Шмидта. Внесем изменения в условия п. 1:

a) параметр $\lambda \in \mathbb{R}$;

b) частоты $\omega_{1}$ и $\omega_{2}$ несоизмеримы;

c) отказьваемся от гипотезы Н4.

Пусть $\left\{\varphi_{0}, \varphi_{1}, \varphi_{2}\right\},\left\{L_{0}, \Phi_{1}, \Phi_{2}\right\}$ - собственные векторы операторов $A_{0}, A_{0}^{*}$ соответственно: $A_{0} \varphi_{0}=0, A_{0} \varphi_{k}=i \omega_{k} \varphi_{k}, A_{0}^{*} \Phi_{k}=-i \omega_{k} \Phi_{k},\left(\varphi_{0}, L_{0}\right)=1,\left(\varphi_{k}, \Phi_{k}\right)=1, k=1,2$.

Введем обозначения: $\widehat{H}=L_{2}\left(T^{2}, H\right)$ - гильбертово пространство вектор-функций на торе $T^{2}=S^{1} \times S^{1}$ со значениями в $H ; \tau=\left(\tau_{1}, \tau_{2}\right)$ - угловые переменные на торе, a $\widehat{H}^{0}$ - подпространство в $\widehat{H}$, состоящее из вектор-функций вида: $u(\tau)=\beta \varphi_{0}+\sum_{k=1}^{2}\left(\alpha_{k} \varphi_{k} e^{i \tau_{k}}+\alpha_{k}^{*} \varphi_{k}^{*} e^{-i \tau_{k}}\right)$, где $\alpha_{k} \in C, \beta \in \mathbb{R}$ - произвольные постоянные. Определим проектор П: $\widehat{H} \rightarrow \widehat{H}^{0}$ :

$$
(\Pi f)(\tau)=2 \sum_{k=1}^{2} \operatorname{Re}\left[\left\langle\left(f, \Phi_{k}\right) e^{-i \tau_{k}}\right\rangle e^{i \tau_{k}} \varphi_{k}\right]+\left(\langle f\rangle, L_{0}\right) \varphi_{0},\langle f\rangle \stackrel{\text { def }}{=} \int_{T^{2}} f(\tau) d \tau .
$$

Через $\widehat{H}^{\prime}$ обозначим дополнительное подпространство, а через $\Pi^{\prime}$ - дополнительный проектор, так что $\widehat{H}=\widehat{H}^{0} \oplus \widehat{H}^{\prime}, \Pi^{\prime}=I-\Pi$.

Введем оператор $R: R u=\omega_{1} \partial u / \partial \tau_{1}+\omega_{2} \partial u / \partial \tau_{2}-A_{0} u\left(\tau_{k}=\omega_{k} t, k=1,2\right)$ с областью определения $\mathscr{D}(R)$, состоящей из вектор-функций, допускающих сильные производные $\partial u / \partial \tau_{i} \in$ $\widehat{H}^{\prime}$. Его сужение $R_{1}$ на $\widehat{H}^{\prime} \cap \mathscr{D}(R)$ - обратимый оператор.

Пусть $\mathscr{A}=\left(a_{i j}\right)_{i, j=1}^{3}-$ матрица, задаваемая выражениями

$$
a_{k k}=\operatorname{Re} g_{k}, \quad a_{k 3}=\operatorname{Re} \eta_{k}, \quad a_{12}=\operatorname{Re} \xi_{12}, \quad a_{21}=\operatorname{Re} \xi_{21}, \quad a_{3 k}=\left(F_{0}^{\prime \prime}\left(\varphi_{k}, \varphi_{k}^{*}\right), L_{0}\right), \quad a_{33}=0
$$

$$
\begin{gathered}
g_{k}=\left\langle\left(F_{0}^{\prime \prime}\left(\psi_{k}, v_{k}\right)+\frac{1}{6} F_{0}^{\prime \prime \prime} \psi_{k}^{3}, \Phi_{k}\right) e^{-i \tau_{k}}\right\rangle, \eta_{k}=\left(F_{0}^{\prime \prime}\left(\varphi_{k}, \varphi_{0}\right), \Phi_{k}\right), \\
\xi_{k j}=\left\langle\left(F_{0}^{\prime \prime}\left(\psi_{k}, v_{j}\right)+F_{0}^{\prime \prime}\left(\psi_{j}, v_{3}\right)+\frac{1}{2} F_{0}^{\prime \prime \prime}\left(\psi_{1}^{k}, \psi_{2}^{j}\right), \Phi_{k}\right) e^{-i \tau_{k}}\right\rangle
\end{gathered}
$$

где $k=1,2$, функция $\psi_{k}\left(\tau_{k}\right)=\varphi_{k} e^{i \tau_{k}}+\varphi_{k}^{*} e^{-i \tau_{k}}$, a $v_{1}, v_{2}, v_{3} \in \widehat{H}^{\prime}$ :

$$
v_{k}=\frac{1}{2} R_{1}^{-1} \Pi^{\prime} F_{0}^{\prime \prime} \psi_{k}^{2}, \quad k=1,2, \quad v_{3}=R_{1}^{-1} \Pi^{\prime} F_{0}^{\prime \prime}\left(\psi_{1}, \psi_{2}\right) .
$$

Здесь приняты обозначения $F_{0}^{\prime}=F_{\theta}^{\prime}\left(\theta_{0}, \lambda_{0}\right), F_{0}^{\prime \prime}=F_{\theta}^{\prime \prime}\left(\theta_{0}, \lambda_{0}\right), \ldots$

Далее понадобятся определители $\Delta=\operatorname{det} \mathscr{A}, \Delta_{1}=a_{23} \operatorname{Re} \zeta_{1}-a_{13} \operatorname{Re} \zeta_{2}, \Delta_{2}=\operatorname{det} \mathscr{A}_{2}$. Матрица $\mathscr{A}_{2}$ получается заменой 3 -й колонки матрицы $\mathscr{A}$ на колонку $\left(-\operatorname{Re} \zeta_{1},-\operatorname{Re} \zeta_{2}, 0\right)$;

$$
\zeta_{k}=\left(F_{0}^{\prime \prime}\left(\varphi_{k}, v_{0}\right)+F_{0 \lambda}^{\prime} \varphi_{k}, \Phi_{k}\right), \quad v_{0}=R_{1}^{-1} F_{0 \lambda}, \quad k=1,2 .
$$

Применение метода Ляпунова-Шмидта приводит к следующей теореме, в которой пункты 2) и 3) сформулированы с точностью до утверждения о сходимости формальных степенных рядов. 
ТЕОРемА. Пусть для уравнения (1) с правой частью $F$, аналитической в окрестности точки $\left(\theta_{0}, \lambda_{0}\right)$, где $\left(\theta_{0}, \lambda_{0}\right)$ - равновесие: $\boldsymbol{F}\left(\theta_{0}, \lambda_{0}\right)=0$, и с косимметрией $\mathscr{L}$ выполнень зипотезы Н1-H3 n. 1 и предположсения а), b) $n .2$.

1) Если выполняется неравенство $a_{31} a_{32} \neq 0 \quad\left(a_{31} a_{32}>0\right)$, то при малых $\delta$ в окрестности $\theta_{0}$ нет мальх предельных ииклов (торов).

2) Пусть выполнены неравенства $a_{31} a_{32}<0 u \Delta_{1} \neq 0$. Тогда $\lambda_{0}$ есть точка ответвления тора. При этом имеются лишь две возможности: либо существует единственный нормальный (вектор частот $\left(\nu_{1}, \nu_{2}\right)$ при $\lambda \rightarrow \lambda_{0}$ стремится $\left.\kappa\left(\omega_{1}, \omega_{2}\right)\right)$ тор, и эта бифуркация односторонняя, либо при $\lambda=\lambda_{0}$ уравнение (1) имеет однопараметрическое семейство квазипериодических двухчастотных решений $\left\{\theta_{\alpha}\right\}$, представимое формальним разлохсением по параметру $\alpha$ ( $\theta_{\alpha}$ не является равновесием при $\left.\alpha \neq 0\right)$. Если при этом $\delta=\lambda-\lambda_{0}$ достаточно мало, но отлично от нуля, то уравнение (1) не имеет малых квазипериодических двухчастотных решений, не являющ,ихся равновесными.

3) Пусть справедливы неравенства $a_{31} a_{32}<0, \Delta \neq 0, \Delta_{1} \neq 0$. Тогда для малого тора, стягивающегося при $\lambda \rightarrow \lambda_{0} \kappa$ равновесию $\theta_{0}$, справедливо формальное асимптотическое разлохение

$$
\theta(t)=\theta_{0}+\sqrt{\gamma_{1} \delta} \psi_{1}\left(\omega_{1} t\right)+\sqrt{\gamma_{2} \delta} \psi_{2}\left(\omega_{2} t\right)+\delta\left(\gamma \varphi_{0}+v_{0}+\gamma_{1} v_{1}+\gamma_{2} v_{2}+\sqrt{\gamma_{1} \gamma_{2}} v_{3}\right)+\cdots
$$

әде опущены члены порядка $\delta^{3 / 2}$ и выше, а амплитудные коэффициенты $\gamma, \gamma_{k}$ задаются равенствами: $\gamma=\Delta_{2} / \Delta, \gamma_{1}=a_{31} \Delta_{1} / \Delta, \gamma_{2}=-a_{32} \Delta_{1} / \Delta$. Это решение вещественно либо при малых $\delta>0$, если $\gamma_{1}>0$, либо при $\delta<0$, когда $\gamma_{1}<0$.

Частоты $\nu_{k}, k=1,2$, этого тора зависят от $\delta$ и имеют вид

$$
\nu_{k}=\omega_{k}+\operatorname{Im}\left(\zeta_{k}+\gamma \eta_{k}+\gamma_{k} g_{k}+\mu_{k}\right) \delta+\cdots, \quad \mu_{1}=\gamma_{2} \xi_{12}, \quad \mu_{2}=\gamma_{1} \xi_{21}
$$

Хорошо известно, что при исследовании квазипериодических режимов и их возмущений возникают трудные проблемы, связанные с малыми знаменателями [4]-[6]. В различных формах эта трудность возникала и, в известной мере, преодолена и в проблеме сохранения квазипериодических режимов при возмущении гамильтоновых систем (КАМ теория), и при изучении бифуркаций вынужденных и автоколебательных периодических режимов, связанных с колебательной неустойчивостью и возникновением новой частоты [7], [8] и при исследовании инвариантных кривых отображения (к этой задаче сводится предыдущая, когда рассматривается оператор монодромии или отображение Пуанкаре), и при исследовании ответвления инвариантного тора от равновесия, имеющего в составе своего спектра две пары чисто мнимых собственных значений (эта бифуркация коразмерности 2 была исследована в работах [9], [10]).

Имеющиеся в этой области результаты подразделяются на две категории. $\mathrm{K}$ первой отнесем утверждения о существовании двумерных инвариантных торов без указания на характер динамики сужения системы на такого рода тор, а ко второй - результаты о существовании инвариантных торов, на которых движения квазипериодические.

Во всех упомянутых исследованиях существенную роль играют резонансные соотношения вида $m_{1} \omega_{1}+m_{2} \omega_{2}=0, m_{1}, m_{2} \in \mathbb{Z}$. Каждый раз, чтобы прийти к определенному результату, приходится предполагать, что либо все эти резонансные соотношения не вьполняются, так что отношение $\omega_{1} / \omega_{2}$ иррационально (так и п. 2 данной статьи), либо, по крайней мере, не выполнены резонансные соотношения с малыми $m_{1}, m_{2}$ (см. п. 1 ). 
Если бы отношение $\omega_{1} / \omega_{2}$ было рациональным, то удалось бы построить лишш конечное число членов рядов (5) и (6). Мы не занимаемся здесь доказательством их сходимости. Его можно провести, накладывая на соотношения $\omega_{1} / \omega_{2}$ обычные арифметические ограничения плохой аппроксимируемости рациональными дробями [6], [11], [12].

\section{СПИСОК ЦИТИРОВАННОЙ ЛИТЕРАТУРЫ}

1. Куракин Л. Г., Юдович В. И. // Матем. заметки. 1999. Т. 66. № 2. С. 317-320. 2. Кurakin L. G., Yudovich V. I. // Chaos. 2000. V. 10. № 2. Р. 311-331. 3. Куракин Л. Г., Юдович В. И. // Докл. РАН. 2000. Т. 372. №1. С. 29-33. 4. Арнольд В. И. и др. Теория бифуркаций // Итоги науки и техники. Современные проблемы математики. Фундаментальные направления. Т. 5.. М.: ВИНИТИ 1986. С. 5-220. 5. Guckenheimer J. // SIAM J. Math. Anal. 1984. V. 15. №1. P. 1-49. 6. Iooss G. Bifurcation of maps and applications. North-Holland Mathematics Studies № 36. Amsterdam-New York-Oxford: North-Holland, 1979. 7. Sacker R. J. On invariant surfaces and bifurcation of periodic solutions of ordinary differential equations / New York Univ. IMM-NYU. 1964. № 333. 8. Ruelle O., Takens F. // Comm. Maths. Phis. 1971. V. 20. P. 167-192. 9. KKoлондек Х. // УМН. 1986. Т. 41. № 5(251). C. 175-176. 10. Jost R. and Zender E. // Helvetica Physica Acta. 1972. V. 45. P. 258-276. 11. Колмогоров А. Н. // Докл. АН СССР. 1954. Т. 98. № 4. C. 527-530. 12. Herman M. Mesure de Lebesgue et nombre de rotation. Geometry and topology. Lecture Notes in Matematics. V. 597. New York: Springer-Verlag, 1977. 